\title{
Photon Acceleration-Based Radiation Sources
}

\author{
J. R. Hoffman, P. Muggli, T. Katsouleas, W. B. Mori*, and C. Joshi*
}

Lnversity of Southern California. Los Angeles, (.4 90089-1)484

* Liniversiby of California ar Los Angeles, l.os Angeles. C. 90095

\begin{abstract}
The acceleration and deceleration of photons in a plasma provides the means for a series of new radiation sources. Previous work on a DC to AC Radiation Converter (DARC source) has shown variable accelcration of photons having zero frequency (i.e., an electrostatic field) to between 6 and $100 \mathrm{GHz}(1-3)$. These sources all had poor guiding characteristics resulting in poor power coupling from the source to the load. Continuing research has identified a novel way to integrate the DARC source into a waveguide. The so called "pin structure" uses stainless steel pins inserted through the narrow side of an X band waveguide to form the electrostatic field pattern $(k \neq 0,(1)=0)$. The pins are spaced such that the absorption band resulting from this additional periodic structure is outside of the $X$ band range $(8-12 \mathrm{GHz})$, in which the normal waveguide characteristics are left unchanged. The power of this $X$ band source is predicted theoretically to scale quadratically with the pin bias voltage as $-800 \mathrm{~W} /(\mathrm{kV})^{2}$ and have a pulse width of - Ins. Cold tests and experimental results are presented. Applications for a high power. short pulse radiation source extends to the areas of landmine detection, improved radar resolution. and experimental investigations of molecular systems
\end{abstract}

\section{INTRODUCTION}

Three methods of photon acceleration have been proposed in the past: the photon accelerator. which uses a plasma wakefield to accelerate a photon bunch: frequency upshifting by over and underdense ionization fronts; and DC to AC radiation converters (see figure 1). In the first scheme called the photon accelerator. an initial laser pulse moves through a plasma producing a plasma wake which accelerates a trailing laser pulse. The amount of acceleration. or equivalently. the frequency upshift of trailing photons is given by

$$
\omega_{1}=\omega_{1}\left[1+2\left(2 \varepsilon^{1 / 2}\right)+4 \varepsilon\right] \text {. }
$$

where $(1)_{1}$ is the frequency of the accelerated photon. $(1)$ is the frequency of the incident photon. and $\varepsilon$ is the plasma wave amplitude (typically, $0.1-0.5$ ). For typical values of $\varepsilon$. an upshift factor of about 5 can be achieved(4).

In the second scheme. a traveling electromagnetic wave is incident upon a moving ionization front (plasma/gas boundary usually produced by a laser), and is either reflected. if the plasma density is such that the corresponding plasma frequency is larger than the double Doppler shifted frequency (overdense). or is transmitted. if the plasma density is below the transmitted frequency (underdense). The frequency

CP472, Advanced Accelerator Concepts: Eighth Workshop, edited by W. Lawson, C. Bellamy, and D. Brosius

(C) 1999 The American Institute of Physics 1-56396-889-4/99/\$15.00 


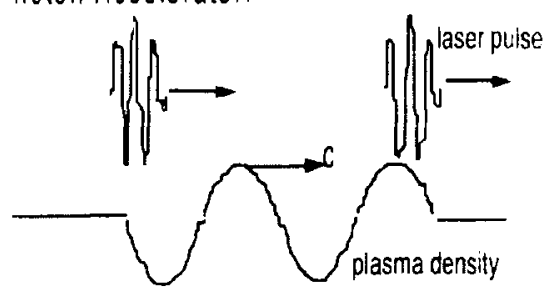

B) Ionization Fronts:

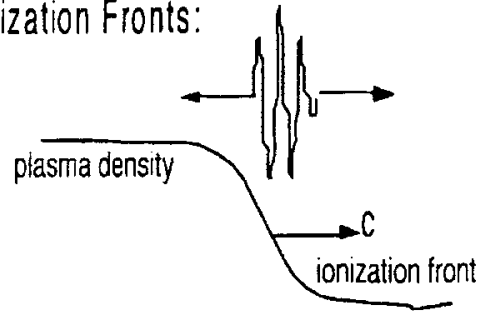

\section{C) $D C$ to $A C$}

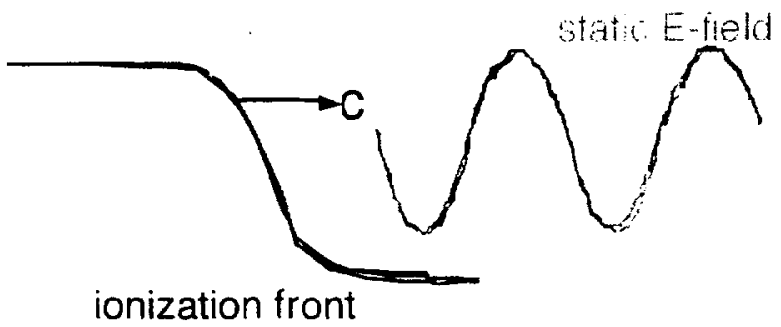

FIGURE 1. Three schemes for accelerating photons. a) the photon accelerator; b) the moving ionization front; and $\mathrm{c}$ ) the $\mathrm{DC}$ to $\mathrm{AC}$ converter.

upshift of the transmitted wave in the underdense case is given by

$$
\omega_{1}=\omega_{1}\left[1+\left(\omega_{p} / 2\left(\omega_{1}\right)^{2}\right]\right. \text {. }
$$

where $\omega_{\mathrm{p}}$ is the plasma frequency. For a plasma density of $4 \times 10^{15} \mathrm{~cm}^{-3}$ and an incident frequency of $33 \mathrm{GHz}$. the predicted upshifted frequency is $3 \mathrm{THz}$. (i.e., an upshift factor of a 100)(5).

In the last scheme, a static electric field is frequency upshifted from zero frequency to

$$
(1)_{t}=k_{0} v_{p} / 2+(1)_{p}^{2} / 2 k_{0} v_{f} \text {. }
$$

where $k_{0}$ is the wavenumber of the static field $\left(k_{t}=\pi / d\right)$. where $d$ is the distance between the capacitor plates. and $v_{f}$ is the velocity of the front. For a static wavelength of $2 \mathrm{~cm}$ and a plasma density of $6 \times 10^{12} \mathrm{~cm}^{-3}$. the predicted transmitted wave frequency is $15 \mathrm{GHz}(6)$. Of the three schemes, the last is the easiest to realize in the laboratory. since only one laser and DC power supply are needed. In the DARC source or. DC to AC converter, alternately biased capacitors create a position dependent $\left(k_{\mathrm{n}} \neq 0\right)$ static electric field. In the front frame, this static field approximates an electromagnetic wave with a frequency $\gamma \mathrm{k}_{\mathrm{i}} \mathrm{v}_{\mathrm{i}}$. where $\gamma$ is the Lorentz factor $1 /(1-$ $\left.\left(v_{1} / c\right)^{2}\right)^{1 / 2}$. For the case of a sharp front. the amplitude of the transmission coefficient is approximately 1(6). (Note. in the ionization front case, there are four transmitted and one reflected wave. all with different frequencies). Of practical interest. the laser 
energy is just used to make the moving ionization front. and does not contribute to the energy of the transmitted waves.

The theoretical power in the transmitted wave of interest is:

$$
\mathrm{P}=\mathrm{E}_{\mathrm{o}}{ }^{2} \mathrm{~V}_{\text {group }} \mathrm{A} / 8 \pi \text {. }
$$

where $E_{0}$ is the amplitude of the first fundamental $y$ component of the electric field butween the plates. $V_{\text {group }}$ is the group velocity of the transmitted wave. and $A$ is the transverse area of the plasma. For parallel plate capacitors. $E_{0} \alpha V_{0} / b$. where $V_{0}$ is the voltage applied to the capacitors, $b$ is the separation between the plates. and thus. the power is proportional to

$$
P \alpha\left(V_{0} / b\right)^{2} A V_{\text {groun. }}
$$

For the DARC structure shown in figure $3 \mathrm{a}$, the theoretically predicted power scaling is $250 \mathrm{~W} / \mathrm{kV}^{7}$, whereas, experimental measurements(2) showed a power scaling of $100 \mathrm{~mW} / \mathrm{kV}^{2}$. This discrepancy will be discussed further in a later section.

Consider some different examples of DARC sources shown in figure 2. The first picture. 2a shows the proof-of-principle structure(2), that is a series of alternatively biased parallel plate capacitors. The second picture. $2 \mathrm{~b}$ shows a Ka band waveguide structure with continuous sidewalls and sectioned top and bottom walls(3). The third picture. $2 \mathrm{c}$ is of a coaxial structure sectioned perpendicularly to the axis. Common to all of these structures are transversc cuts relative to the direction of propagation of the transmitted wave. In order to support the well-known TE. TM. or TEM modes of these structures. the structure has to be able to support the wall currents. A cold test of the $\mathrm{Ka}$ band structure (figure 2b) showed a $30 \mathrm{db}$ loss in transmission. The conclusion to be drawn

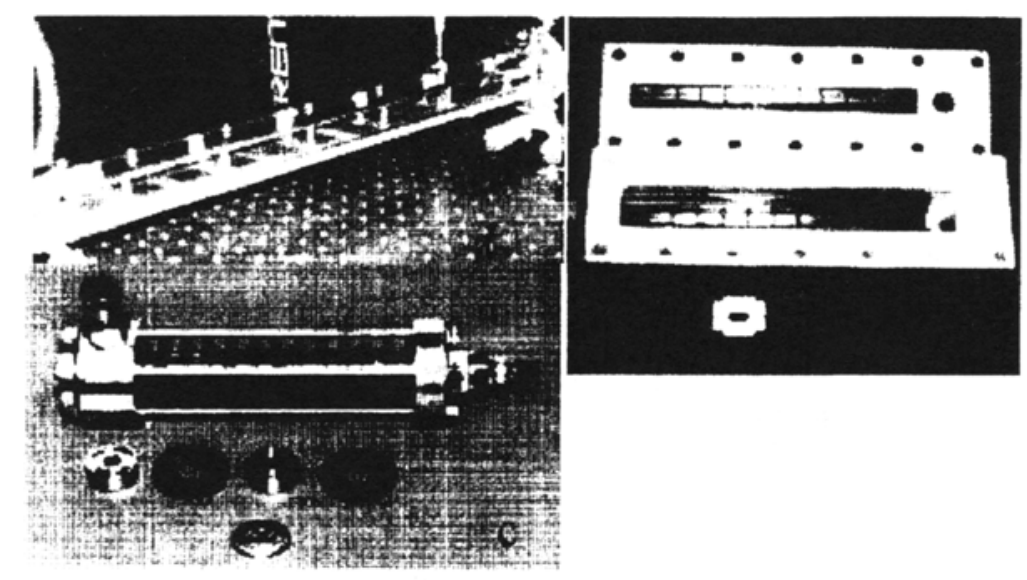

FIGURE 2. a) Proof of principle DARC source, 12 sets of parallel plate; b) Ka band DARC source, a transversely sectioned Ka waveguide; c) Coaxial DARC source, a transversely sectioned coax line. 
from this is that sectioning of the waveguide is not an acceptable way to produce the static electric field.

A new idea is proposed here to produce the static field: insert pins through the short side of the waveguide, thus minimizing the perturbation to the continuity of the waveguide walls (figure 3 ). The pins form a periodic structure inside of the waveguide with stopband frequencies given by :

$$
(1)_{\mathrm{sh}}=\mathrm{c}\left[(\pi / \mathrm{h})^{2}+(1 \pi / \mathrm{d})^{2}\right]^{1 / 2} \text {. }
$$

where $\mathrm{c}$ is the speed of light, $\mathrm{h}$ is the vertical spacing between the pins, $\mathrm{d}$ is the longitudinal spacing between the pins, and $l$ is the mode number. By judicious selection of the spacing (d) between the pins, these stopband frequencies can be placed

a)
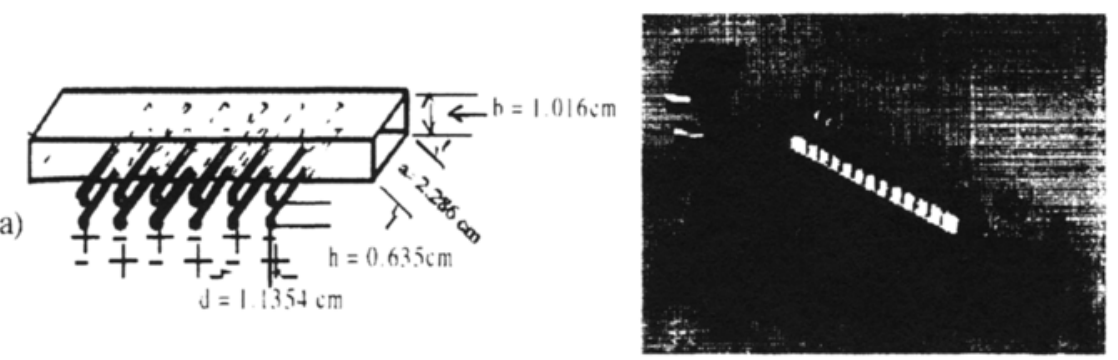

FIGURE 3. a) a dimensional cartoon of an $X$-band waveguide showing where the pin electrodes are inserted. b) the actual implementation of the $X$-band pin structure.

outside of the $\mathrm{X}$ band frequency range. For an $\mathrm{X}$-band waveguide, with $\mathrm{h}=0.635 \mathrm{~cm}$. and $d=1.1354 \mathrm{~cm}$. the first stopband $\omega_{s b}(l=1)$ corresponds to a frequency of 14.6 $\mathrm{GHz}$, and the transmission characteristics of the fundamental $\mathrm{TE}_{10}$ mode of the waveguide should remain unchanged between 8 and $12 \mathrm{GHz}$. The transmission characteristics of the pin structure was measured with an HP network analyzer. model $8720 \mathrm{~A}$. and compared to an ordinary waveguide. These results are shown in figure 4 .

\section{PIN POWER AND PULSEWIDTH CALCULATIONS}

Figure 5 shows a comparison of the potential and $y$ component of the electric fields of the pins and parallel plates at the same separation. $h=0.663 \mathrm{~cm}$, and period $d$ $=1.135 \mathrm{~cm}$. The $y$ component of the electric field of the pins is well concentrated between the pins and falls off rapidly to zero in the void separating the pins. Whereas. in the parallel plate case, the electric field fills most of the space between the plates and has a fairly constant amplitude. The reduction factor in the expected power due to the different electric field configurations is currently under investigation. and will be reported on in a later paper.

The expected pulse width can be approximated by 


$$
\tau=\mathrm{Nc} / \lambda=\mathrm{N} / \mathrm{f},
$$

where $N$ is the number of cycles (i.e.. the number of pairs of pins divided by two), $c$ is the speed of light, $\lambda$ is the wavelength of the transmitted wave. Assuming an output frequency of $10 \mathrm{GHz}$, the expected pulse width is $1.2 \mathrm{~ns}$ for $\mathrm{N}=6$.

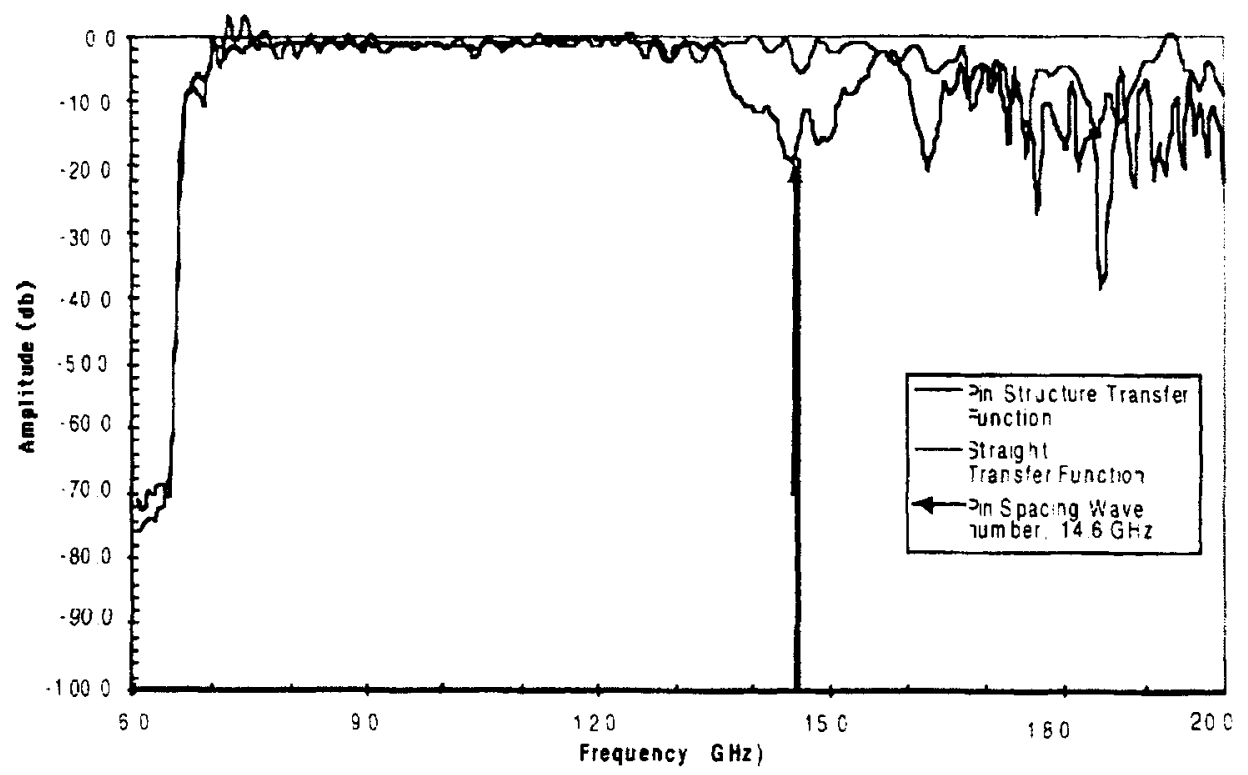

FIGURE 4. The $S_{12}$ transmission characteristics of the $X$-band pin structure compared to a standard $X$-band waveguide. The vertical arrow points to the theoretical position of the periodic reflection due to the inserted pin.

\section{EXPERIMENT}

The experiment was performed at the UCLA Neptune lab facility using a frequency quadrupled YAG laser beam with a pulsewidth of $50 \mathrm{ps}$, a wavelength of $266 \mathrm{~nm}$, and an energy of $\sim 27 \mathrm{~mJ}$. The ionizable gas was a volatile organic compound called TMAE, and the pressure inside of the pin structure was maintained at $\sim 50 \mathrm{mT}$. During the experiment the shot to shot variation of the laser energy was observed to be \pm 7 percent. The pressure drift was no more than $\pm 5 \%$.

The microwave detection diode was calibrated using a microwave power meter and source (figure 6). As can be seen from the insert on the lower right side of figure 6 . the diode saturates at relatively low power levels. approximately $0.4 \mathrm{~W}$. The detection diode was calibrated up to $1 \mathrm{~W}$ of power. and a power law fit was made to this data. The power produced in the pin structure was inferred from the detection diode signal 
Pin nerentive
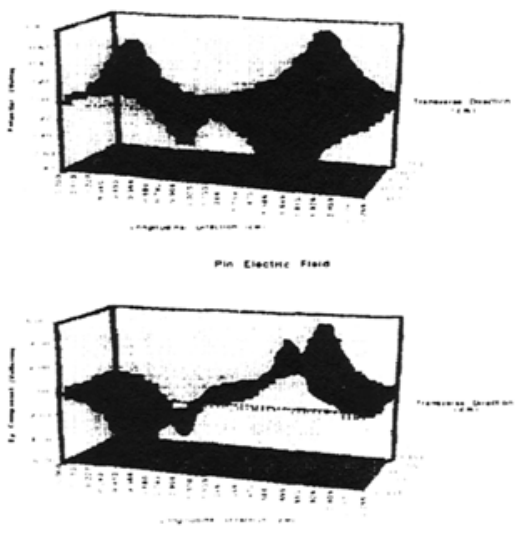

note netertion

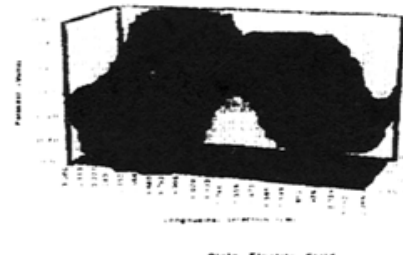

piote tioctirt riend

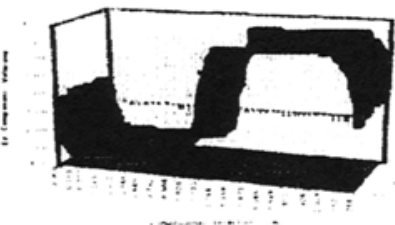

FIGURE 5. Plots of the analytical solutions of the potential and electric fields: a) 3D contour plot of the scalar potential between two sets of pins. b) 3D contour plot of the y component electric field between two sets of pins. c) 3D contour plot of the scalar potential between two sets of plates. d) 3D contour plot of the y component of the electric field between two sets of plates.

\section{Microwave Diode Signal vs Power}

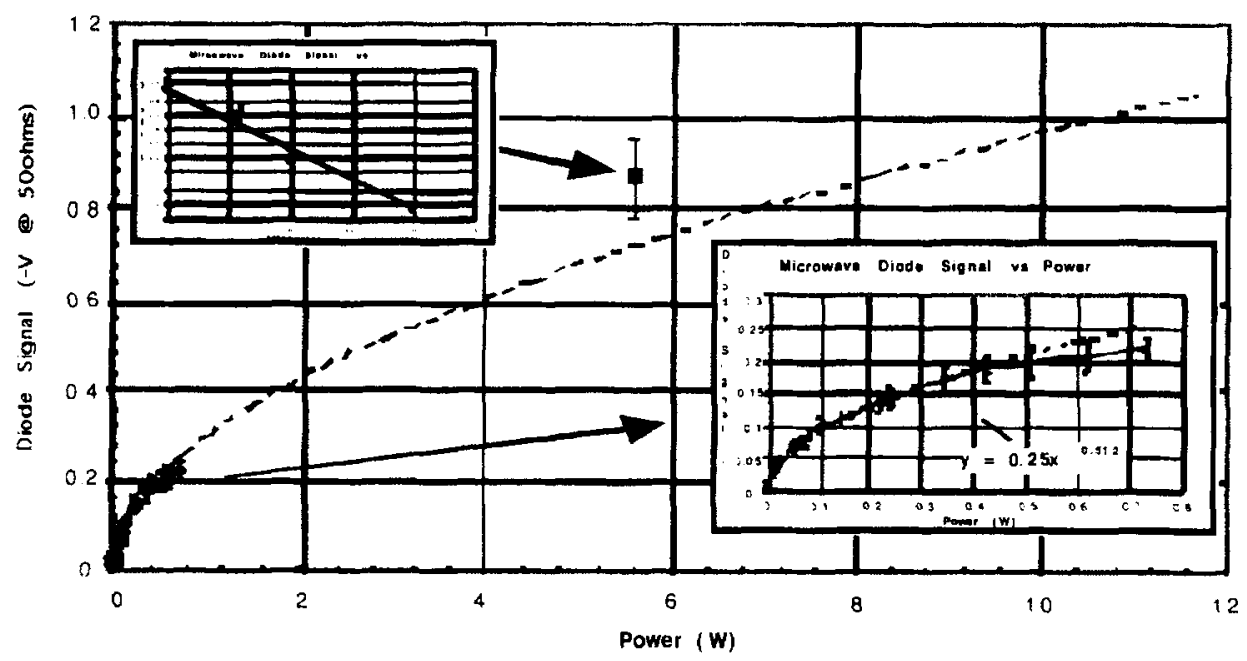

FIGURE 6. The microwave detection diode calibration results. The bottom right insert shows the measured diode voltage as a function of input power. The dotted line curve in the insert and in the main figure is a power curve fit to the measured data. The top left insert shows data points taken using the pin structure as a power source and placing various attenuators between the pin structure and the detection diode. 
by using the extrapolated power law curve of figure 6 . The upper left insert in figure 6 shows data from the experiment where an attenuator was used on the output signal. This was done in an attempt to get a calibration of the detection diode at higher power levels. The result is the point on the main graph indicated by the arrow from the upper insert.

\section{EXPERIMENTAL RESULTS}

Figure 7 shows the measured microwave diode signal plotted versus applied voltage squared. The unsaturated data points (power less than $5 \mathrm{~W}$ ) are indicated by the diamonds. and the saturated data points are indicated by the squares. $A$ least squared fit line drawn through the unsaturated points yields a slope of $\sim 1 \mathrm{~W} / \mathrm{kV}^{2}$. The microwave diode also showed a response time of $\sim 4$ ns. roughly a factor of 4 above the predicted pulse width (again, assuming an output frequency of $10 \mathrm{GH}$ ). Since the diode acts like an integrator. we estimate that the actual peak power for a 1 ns pulse is a lactor of four greater, or $\sim 4 \mathrm{~W} / \mathrm{kV}^{2}$. For our peak applied voltage of $7 \mathrm{kV}$. we infer the peak power to be $\sim 200 \mathrm{~W}$. This is much larger than the powers observed in either of the previous DARC structures. Further work is planned to improve the power diagnostic and to obtain theoretical power predictions for the pin structure.

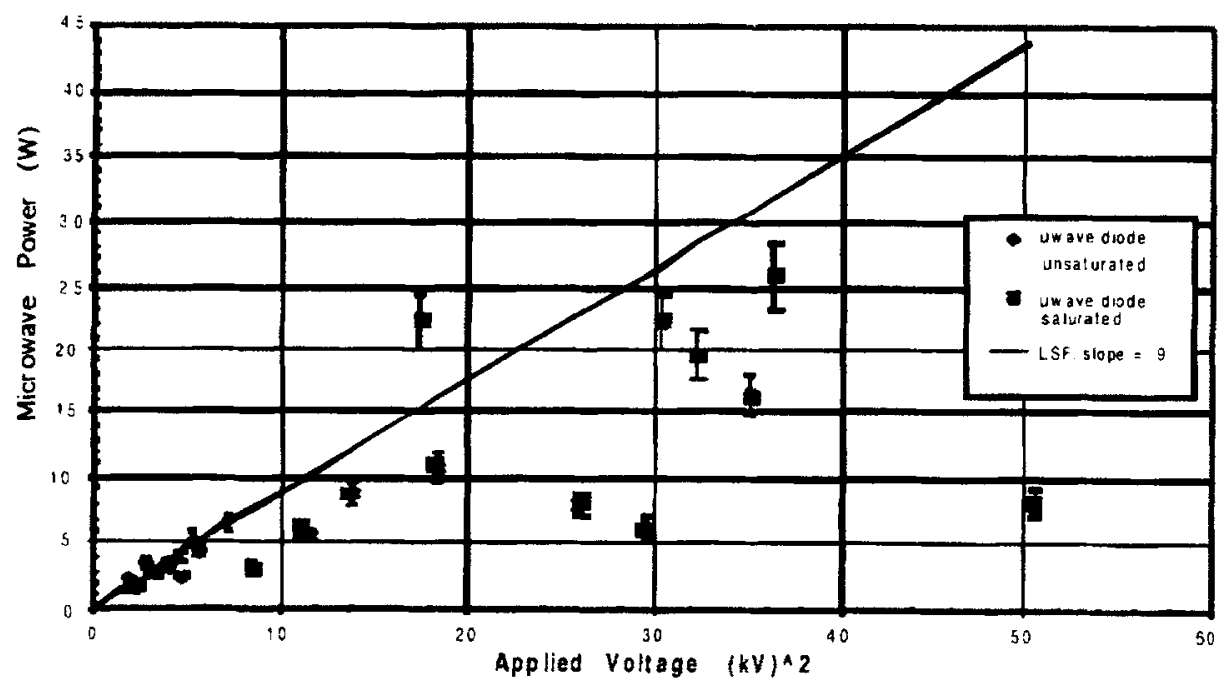

FIGLiRE 7. The measured microwave power versus applied voltage squared. The square data points are the data points we believe the microwave diode was saturated, so a least squared fit (LSF) was calculated only through the diamond points. 


\section{CONCLUSION}

In conclusion, the novel pin structure produced power that is inferred to be in the hundred Watts range. a large improvement with respect to other DARC sources that reached power levels of milliwatts. Future cxperiments will try to measure the frequency spectrum, and the feasibility of detecting buried objects (e.g.. landmines) inthe ground with this ultrashort putse microwave source.

\section{ACKNOWLEDGEMENTS}

This work is supported by the National Science Foundation, Grant No. ECS-9632735: the Air Force Office for Scientific Research. Grant No. F49620-95-1-0248: and the US Department of Energy, Grant Vo. DE-FG03-92ER-40745.

\section{REFERENCES}

1. Savage, Jr.. R. L., Brogle, R. P., Mori et al.. "Frequency upshifting and pulse compression via underdense relativistic ionization fronts." IEEE: Transactions on Plasma Science 21 (1). $5-19(1993)$.

2. Lai. C. H., Liou. R., Katsouleas. T. C., et al., "Dentonstration of microwave generation from a static field by a relativistic ionization front in a capacitor array." Physical Review Letters 77 (23). 4764-7 (1996).

3. Muggli, P.. Liou. R.. Lai, C. H., et al., "Generation of microwave pulses from the static electric field of a capacitor array by an underdense, relativistic ionization front." Physics of Plasmas5 (5). 2112$19(1997)$

4. Wilks, S. C. Dawson, J. M.. Mori. W. B., et al.. "Photon accelerator." Physical Review Letters $62(22) .2600-3(1989)$.

5. Mori, W. B., "Generation of tunable radiation using an underdense ionization front," Physical Review A 44 (8), $5118-21$ (1991).

6. Mori, W. B. , Katsouleas. T. Dawson. J. M. er al., "Conversion of de fields in a capacitor array to radiation by a relativistic ionization front." Physical Review Letters 74 (4), 542.5 (1995) 